Published in final edited form as:

Curr Opin Neurol. 2015 June ; 28(3): 237-243. doi:10.1097/WCO.0000000000000195.

\title{
Progressive multiple sclerosis
}

Daniel Ontaneda, MD, MS and Robert J. Fox, MD, MS

Mellen Center for Multiple Sclerosis, Cleveland Clinic Lerner College of Medicine

\begin{abstract}
Purpose to Review-To highlight the pathological features and clinical aspects of progressive multiple sclerosis (PMS). To highlight results of clinical trial experience to date and review ongoing clinical trials and perspective new treatment options. Explain the challenges of clinical trial design in PMS.
\end{abstract}

Recent Findings-MS has been identified as a chronic immune mediated disease, and the progressive phase of the disease appears to have significant neurodegenerative mechanisms. The classification of the course of PMS has been re-organized into categories of active vs. inactive inflammatory disease and the presence vs. absence of gradual disease progression. This differentiation allows clearer conceptualization of PMS and possibly even more efficient recruitment of PMS subjects into clinical trials. Clinical trial experience to date in PMS has been negative with anti-inflammatory medications used in relapsing MS. Simvastatin was recently tested in a phase II trial and showed a $43 \%$ reduction on annualized atrophy progression in secondary progressive MS. Ongoing PMS trials are currently being conducted with the phosphodiesterase inhibitor ibudilast, S1P modulator siponimod, and anti-B-cell therapy ocrelizumab. Several efforts for development of outcome measures in PMS are ongoing.

Summary-PMS represents a significant challenge, as the pathogenesis of the disease is not well understood, no validated outcome metrics have been established, and clinical trial experience to date has been disappointing. Advances in the understanding of the disease and lessons learned in previous clinical trials are paving the way for successful development of disease modifying agents for this disease.

\section{Keywords}

Multiple sclerosis; Secondary progressive; Primary progressive; Clinical trials

\section{Introduction}

Progressive multiple sclerosis (PMS) is a clinical form of MS characterized by gradual accrual of disability independent of relapses over time. Secondary progressive MS (SPMS)

Corresponding Author: Daniel Ontaneda MD MS, Mellen Center for Multiple Sclerosis Treatment and Research Cleveland Clinic Foundation, 9500 Euclid Avenue, U-10, Cleveland, OH, USA ontaned@ccf.org Tel: 216-444-0151. Fax 216-445-6259.

Conflicts of Interest

Dr. Ontaneda has received consulting or speaking fees from Acorda Therapeutics, Biogen Idec, Genzyme, Malinckrodt and Novartis. Dr. Fox has received grants from the National Multiple Sclerosis Society and National Institutes of Health, as well as consulting fees from Biogen Idec, MedDay, Novartis, Questcor, Teva Neuroscience, and Xenoport, and research support from Biogen Idec and Novartis. 
occurs after an initial relapsing course of the disease and primary progressive (PPMS) occurs with gradual accumulation of disability from the onset. PPMS is the presenting form of the disease in approximately $10 \%$ of patients and a large proportion of subjects with RRMS will eventually go on to develop SPMS. No treatment has been identified to treat purely progressive forms of MS, and this failure is in contrast to the significant advances made in relapsing remitting MS (RRMS), where inflammation can be targeted to modify the disease course. ${ }^{1}$ A significant unmet need exists in the treatment of PMS and this relates to an incomplete understanding of the disease pathogenesis, lack of validated outcome measures and mostly negative clinical trial experience to date. Treatment of PMS with neuroprotective and neurorestorative agents is the next frontier in the journey to provide more complete control of the disease process.

Significant recent advances have been made over the last several years in the understanding of PMS through the development of tools to further our understanding of the basic, clinical and therapeutic mechanisms of the disease. In this paper we will review the main features of PMS and highlight recent advances in PMS.

\section{Pathogenesis}

MS is a chronic inflammatory, demyelinating and neurodegenerative disorder. Inflammation and focal demyelination with break-down of the blood brain barrier are prominent features in relapsing MS. ${ }^{2}$ In PMS focal disruption of the blood brain barrier is less common and widespread degeneration of the white and grey matter with resultant atrophy are pathological hallmarks. ${ }^{3}$ MS may be seen as a spectrum with an intense focal inflammatory component in RRMS and more neurodegenerative features with concomitant chronic inflammation and axon loss in PMS (figure 1). ${ }^{4}$ In PMS focal white matter lesions that accrued earlier in the disease have chronic demyelination and ongoing axonal loss. The underlying pathophysiology of this chronic demyelination and axonal loss is unknown. Potential explanations include dysfunction of oligodendrocytes, astrocytes, microglia, B-cell and humoral immunity, mitochondria, lipids and lipid receptors, and metabolism. ${ }^{5}$ The presence of chronic "active" lesions with a rim of inflammatory cells may also be seen in a subset of PMS patients. ${ }^{6}$ Inflammation may also be seen in PMS as aggregates of inflammatory cells (mainly B-cells) in the meninges which have a follicle like appearance. ${ }^{7}$ Finally the presence of widespread demyelination and lesions in the cortex are common in MS and are a particularly prominent feature in PMS. Compartmentalized inflammation in the meningeal space may relate to the formation of cortical lesions, ${ }^{8}$ although similarly may be a secondary reaction. Indeed, the chronic, bland inflammation seen in PMS may be a secondary response to a different pathologic process, which could explain the previous ineffectiveness of anti-inflammatory therapies in PMS trials. Clearly, the pathogenesis of PMS is not well understood and this is slowing the development of effective therapies.

\section{Clinical Features and Course}

The diagnosis of PMS can be difficult to make when identifying the disease from onset (PPMS) and may go unrecognized by patients or physicians for some time. Similarly, determining when a patient with RRMS has transitioned into SPMS can be difficult since it 
is purely a clinical diagnosis based upon patient history. This difficulty relates in part to the indolent onset of progression of symptoms and to the occasional superimposed clinical relapses in SPMS. Similarly, new lesions or gadolinium enhancing lesions (hallmarks of active inflammation) can be seen during the SPMS phase of the disease. PMS commonly presents as syndromes of neurological progression of one or more symptoms. The most common presentation of PMS is that of a progressive myelopathy, which typically manifests as a spastic, ataxic paraparesis with sphincteric dysfunction. Progressive cerebellar, cognitive, hemiplegic, brainstem syndromes and a combination of each of these may also occur. ${ }^{9}$ When compared with RRMS the diagnosis of PPMS can be delayed. Current diagnostic criteria for PMS require a full year of gradual progression in addition to temporal and anatomic dissemination, which can be supported by cerebrospinal fluid markers. ${ }^{10}$ In a similar way the transition between RRMS and SPMS is not easy to identify and a delay of up to 3 years was recently described in this "transition" period. ${ }^{11}$ This may be due to the fact that differentiating incomplete recovery of relapses and disability may be difficult for patients as well as clinicians. Disability is typically accrued more rapidly in PPMS as compared to SPMS, but it should be noted that relapses may still occur in subjects with SPMS, occasionally even in subjects with PPMS. ${ }^{12}$

Recent changes to the classifications of MS were proposed by an international expert panel to further characterize the clinical course of PMS. ${ }^{13}$ These changes include categorization of disease course in PMS as either having active inflammation (so-called "active") or not having active inflammation (so-called "non-active") based upon the presence of clinical relapses or new T2 lesions.(Figure 2) The expert panel also recommended classifying PMS based on the presence or absence of gradual clinical disease progression. These recommendations relate, in part, to improving the identification of patients for clinical trials, as well as simplifying the designation of PMS. It should be noted that a previous categorization of PMS (progressive relapsing) was abandoned in this more contemporary classification.

\section{Clinical Measures of PMS}

A significant challenge in PMS is the difficulty quantifying disability over time. As outlined above, disability progression in PMS can manifest in many different ways and progression occurs over years. This heterogeneity and slow progression present challenges to measuring disease progression in PMS trials. The Expanded Disability Status Scale (EDSS) is an ordinal scale ranging from 0 to 10 in 0.5 point increments. The EDSS is the most common metric used to define disability progression over time in clinical trials. ${ }^{14} \mathrm{~A}$ worsening of 0.5 or 1 points sustained over $3-6$ months is a common definition of disease progression. The EDSS has several drawbacks including its construct as an ordinal scale, ${ }^{15}$ high inter-rater variability, ${ }^{46}$ over-reliance on lower extremity function, ${ }^{16}$ lack of meaningful cognitive component, ${ }^{17}$ and relative insensitivity to longitudinal change in specific ranges of the scale. ${ }^{18}$ These features have limited the utility of the EDSS both in clinical practice as well as in clinical trials. The Multiple Sclerosis Functional Composite (MSFC) is a quantitative measure of MS related disability and includes arm, leg, visual, and cognitive components. A $20 \%$ change in any MSFC components has been proposed as evidence of disease progression. ${ }^{19}$ The MSFC has its own limitations, as its components are not collected 
routinely in clinical practice and it surveys only a limited amount of neurological function. Furthermore, regulatory acceptance of the MSFC as a primary outcome has been limited. An alternative method for measuring progressive MS, which is easy to conduct, sensitive to change, quantitative, and grounded in patient feel/function is desperately needed. To meet that need, a the Multiple Sclerosis Outcomes Assessment Consortium was developed by the National Multiple Sclerosis Society (USA) and Critical Path Institute to develop and validate a quantitative outcome tool of disability progression that can be used in MS clinical trials. ${ }^{20,21}$ Analysis of existing datasets and technological advances in measuring function are likely to produce improved disability measurement tools over the next several years.

\section{Imaging of Progressive MS}

Magnetic resonance imaging (MRI) is a powerful tool both for the diagnosis of MS and as a potential outcome metric in clinical trials. Demonstration of MS lesions in the brain and spinal cord form part of the diagnostic criteria for MS (including PMS), ${ }^{10}$ allows differentiation from other neurodegenerative diseases, and aids in making a prompt diagnosis of MS. In clinical practice it is difficult to differentiate PMS from relapsing MS using MRI; however studies have found some population-based differences between these two groups. In PMS there tends to be a preponderance of spinal cord/brainstem lesions, relative paucity of brain lesions, and relative paucity of new enhancing brain lesions over time. ${ }^{9}$ However, these characteristics are weak predictors of PMS in individual people with MS. A subset of PPMS patients however do present with enhancing lesions, tend to progress quickly, and may respond to anti-inflammatory treatments. ${ }^{22} \mathrm{~A}$ decrease in the occurrence of new T2 lesions occurs concomitantly to the clinical transition between RRMS and SPMS. A common clinical scenario is a previous relapsing patient who is developing progressive neurological worsening with no change in brain lesion load. MRI spectroscopy studies also show there is greater tissue destruction, as measured by creatine and N-acetylaspartate of lesions in PMS compared to RRMS. ${ }^{23}$ Independent of inflammatory measures brain atrophy is prominent in PMS. ${ }^{24}$ Differences in regional atrophy exist in RR and PPMS. Atrophy in RRMS is mainly due to loss of white matter and can be seen as ventricular enlargement, while in PMS atrophy tends to be more of a cortical phenomenon. ${ }^{25}$ Cortical lesions are more common in PPMS, followed by SPMS and RRMS; this finding may explain in part the increased prevalence of cognitive dysfunction in PMS. ${ }^{26}$

Spinal cord imaging may be especially informative in PMS as this is likely the site of injury as related to the clinical manifestations of the disease. Atrophy of the spinal cord is more prominent in PMS compared to RRMS and is correlated with physical disability. ${ }^{27} \mathrm{~A}$ recent study showed that gray matter atrophy from the spinal cord correlates strongly with disability in PMS and may relate to the primary neurodegenerative aspect of the disease or to atrophy's characterization of the final common results of MS injury. ${ }^{28}$ Measures of tissue injury using diffusion tensor imaging of the spinal cord also show that tissue integrity is lost at greater rates in PMS patients. ${ }^{29}$ Routine spinal cord imaging can also be helpful in excluding other disorders, such as degenerative disc disease.

Optical coherence tomography (OCT) is a non-invasive tool to characterize the retina. OCT measurements of retinal nerve fiber layer thickness and macular volume can be found in 
PMS and correlate with disability. PMS patients tend to have lower retinal nerve fiber layer thickness and lower macular volume than RRMS patients which indicates myelin and axonal loss within the visual system and may be an indicator of both the degree of injury along a spectrum and a predictor of injury in other areas of the central nervous system. ${ }^{30}$

\section{Treatment Options and Clinical Trials}

There are no currently approved treatments for purely progressive forms of MS. Clinical trial experience to date has been mostly disappointing and the identification of a therapy to treat PMS remains elusive. Several ongoing clinical trials with novel therapeutics and outcome measures may identify agents in the not too distant future.

\section{Clinical Trials to Date}

Clinical trial experience in PMS has focused mainly on the use of immune modulating therapies, many of which had shown beneficial effects in RRMS. Phase II/III clinical trials using interferons, ${ }^{31-35}$ glatiramer acetate, ${ }^{36}$ and fingolimod ${ }^{37}$ have shown negative results. Rituximab was tested in PPMS and the primary study endpoints were not met, although a benefit was seen in a subgroup of younger patients with inflammatory brain disease activity at baseline. ${ }^{22}$ Mitoxantrone was shown to be effective in SPMS patients with superimposed relapses (i.e. concomitant infiltrative inflammation similar to that seen in RRMS), but this effect was likely secondary to the infiltrative inflammatory component of SPMS rather than any primary effect on the insidious disease progression aspect of PMS. This explanation is likely similar to the differential benefits of interferon-beta1 in different SPMS trials. ${ }^{38}$ Older immunomodulators, including azathioprine, methotrexate and cyclophosphamide have also been studied and results have not showed a convincing benefit on progression. ${ }^{39-42} \mathrm{~A}$ trial of a myelin basic protein analog (MBP8298) was also found to be ineffective in SPMS. The disappointing results to date of immunomodulators in PMS raise caution about further trials of purely anti-inflammatory therapies in PMS. Although it is possible that chronic, smoldering inflammation may not be adequately suppressed by current immunomodulating therapies, it seems more likely that different pathophysiologic mechanism underlie PMS, making therapies that target more than just the immune system more likely to find success in PMS.

Several potential neuroprotective therapies have been tested in PMS and results have been mixed. Simvastatin at a dose of $80 \mathrm{mg}$ daily was tested in a phase II trial $(n=140)$ in SPMS and showed that simvastatin reduced annualized atrophy rate (the primary outcome) by $43 \%$ compared to placebo. ${ }^{43}$ Simvastatin also showed benefit in both the EDSS as well as well as in the Multiple Sclerosis Impact Scale 29. The results of simvastatin are very promising, although a future phase III trial is challenging given the cost of a phase III MS trial and the absence of patent protection which makes industry development unlikely. Lamotrigine was tested in a phase II study in SPMS but showed no benefit on brain atrophy progression. ${ }^{44}$ The investigators speculated regarding the possible confounding of the anti-inflammatory effects lamotrigine and secondary pseudoatrophy in the first year of the trial, although the validity of that explanation is unclear. A cannabinoid analog dronabinol was tested in a three-year, phase III study, and the results were negative. A lower than expected proportion 
of progression in the study was hypothesized as a possible explanation for the result. ${ }^{45}$ The dronabinol trial highlights the need for shorter, biomarker-driven phase II studies before longer, more expensive phase III studies are carried out. ${ }^{46}$

\section{Ongoing Clinical Trials}

Several anti-inflammatory and putative neuroprotective therapies are currently being studied in PMS (Table 2). Natalizumab, a highly effective monoclonal antibody in RRMS, is currently being tested in a large phase III study in SPMS. The effect of natalizumab on progressive MS will need to be interpreted with caution, given the powerful antiinflammatory actions of the medication and its effects in a SPMS population which may still have inflammatory disease activity. Siponimod is a selective sphingosine 1 phosphate (S1P) modulator and is currently being studied in SPMS. Although the non-selective S1P modulator fingolimod has shown a favorable effect on atrophy in RRMS, the negative results of fingolimod seen in PPMS raises a potential question regarding the efficacy of siponimod in SPMS. Ocrelizumab, a humanized version of rituximab, is being studied in PPMS based on positive results in the subgroup analysis of the rituximab trial. Masitinib is tyrosine kinase inhibitor which showed positive effects in a small phase II study of PMS subjects $^{47}$ and is currently being tested in a phase III study in PPMS and SPMS. Ibudilast is a phosphodiesterase inhibitor which showed a reduction in brain atrophy in a phase II study in RRMS. ${ }^{48}$ Based upon this atrophy benefit in RRMS, ibudilast is currently being studied in a phase II study in SPMS patients in the United States (SPRINT-MS). ${ }^{49}$ The SPRINT MS study will include several advanced imaging metrics including diffusion tensor imaging, cortical thickness, magnetization transfer and optical coherence tomography in all patients at all sites. Regardless of whether ibudilast shows benefit, the SPRINT-MS study will allow a head-to-head comparison of outcome metrics to better guide the conduct of future phase II trials in PMS. The MS-SMART phase II trial will study amiloride, riluzole, and ibudilast compared with placebo in a 4-arm study in the United Kingdom. ${ }^{50}$ The approach of studying multiple active medications at a time allows for minimized exposure to placebo and increases the likelihood of success by studying several medications simultaneously.

Several potential restorative therapies are being studies in early phase trials in PMS. Cell based therapies in the form of stem cells have received significant attention; however a restorative effect has yet to be seen in the trials to date. Potential remyelinating therapies may also be tested in the future.

\section{Challenges and Future Perspectives in Clinical Trials}

Several challenges exist to the development of effective therapeutics in PMS and this relates to an incomplete understanding of the pathogenesis of the disease and to shortcomings in the methodological aspects of clinical trial design. The incomplete understanding of the pathogenesis of PMS and imperfect animal models of PMS ${ }^{51}$ make identification of potential target pathways and agents difficult. Focal inflammatory lesions serve as an effective phase II outcome in RRMS, predicting clinical efficacy in phase III trials with high accuracy. However a similar predictive biomarker does not exist in PMS. A robust, sensitive and grounded clinical outcome for phase III trials is also needed and part of the failures seen 
in phase III trials to date may be due in part to this suboptimal outcome metric. Several innovative clinical trial designs are now being developed and should make clinical trial conduct more efficient and equitable for patients (e.g. minimize exposure to placebo). ${ }^{52}$ The progressive MS Alliance is an international effort to leverage resources across disciplines to further research and treatment development in PMS and holds great promise to help accelerate the development of effective therapies for PMS.. ${ }^{53}$ The Multiple Sclerosis Outcomes Assessment Consortium may provide more refined outcome metrics for use in PMS clinical trials.

\section{Conclusions}

PMS represents an ongoing challenge for scientists trying to understand the disease, clinical researchers attempting to develop therapies, clinicians in treating patients with PMS, and most of all to people with PMS who suffer continued disability. Although previous clinical trials have yielded disappointing results, important lessons have been learned and several promising avenues have been identified and are being pursued. As our understanding of the clinical and basic science around PMS improves, the likelihood of identifying effective therapies is steadily increasing. A concerted effort is being made to develop phase II and phase III outcome metrics to test novel neuroprotective agents in PMS. Many ongoing clinical trials hold great promise for the future.

\section{Acknowledgments}

We would like to thank Sneha Natarajan for technical support.

Financial support and sponsorship

This work was supported by time provided by National Institutes of Health (Clinical and Translational Science Collaborative of Cleveland, (KL2TR000440) KL2 Award to Daniel Ontaneda.

\section{References}

* Special Interest

** Outstanding interest

1. Ontaneda D, Hyland M, Cohen JA. Multiple sclerosis: New insights in pathogenesis and novel therapeutics. Annu Rev Med. 2012; 63:389-404. [PubMed: 21888515]

2. Lucchinetti CF, Brueck W, Rodriguez M, Lassmann H. Multiple sclerosis: Lessons from neuropathology. Semin Neurol. 1998; 18(3):337-349. [PubMed: 9817538]

3. Lassmann H, van Horssen J, Mahad D. Progressive multiple sclerosis: Pathology and pathogenesis. Nat Rev Neurol. 2012; 8(11):647-656. [PubMed: 23007702]

4. Lassmann H. Multiple sclerosis: Is there neurodegeneration independent from inflammation? J Neurol Sci. 2007; 259(1-2):3-6. [PubMed: 17367814]

5. Trapp BD, Nave KA. Multiple sclerosis: An immune or neurodegenerative disorder? Annu Rev Neurosci. 2008; 31:247-269. [PubMed: 18558855]

6. Kornek B, Storch MK, Weissert R, et al. Multiple sclerosis and chronic autoimmune encephalomyelitis: A comparative quantitative study of axonal injury in active, inactive, and remyelinated lesions. Am J Pathol. 2000; 157(1):267-276. [PubMed: 10880396] 
7. Serafini B, Rosicarelli B, Magliozzi R, Stigliano E, Aloisi F. Detection of ectopic B-cell follicles with germinal centers in the meninges of patients with secondary progressive multiple sclerosis. Brain Pathol. 2004; 14(2):164-174. [PubMed: 15193029]

8. Choi SR, Howell OW, Carassiti D, et al. Meningeal inflammation plays a role in the pathology of primary progressive multiple sclerosis. Brain. 2012; 135(Pt 10):2925-2937. [PubMed: 22907116]

9. Stevenson VL, Miller DH, Rovaris M, et al. Primary and transitional progressive MS: A clinical and MRI cross-sectional study. Neurology. 1999; 52(4):839-845. [PubMed: 10078736]

10. Polman CH, Reingold SC, Banwell B, et al. Diagnostic criteria for multiple sclerosis: 2010 revisions to the McDonald criteria. Ann Neurol. 2011; 69(2):292-302. [PubMed: 21387374]

*11. Sand IK, Krieger S, Farrell C, Miller AE. Diagnostic uncertainty during the transition to secondary progressive multiple sclerosis. Mult Scler. 2014; 20(12):1654-1657. Illustrates the difficulty in diagnosis of SPMS. [PubMed: 24493475]

12. Koch MW, Greenfield J, Javizian O, Deighton S, Wall W, Metz LM. The natural history of early versus late disability accumulation in primary progressive MS. J Neurol Neurosurg Psychiatry. 2014

**13. Lublin FD, Reingold SC, Cohen JA, et al. Defining the clinical course of multiple sclerosis: The 2013 revisions. Neurology. 2014; 83(3):278-286. Revised definitions of SPMS which may help recruitment into clinical trials. [PubMed: 24871874]

14. Meyer-Moock S, Feng YS, Maeurer M, Dippel FW, Kohlmann T. Systematic literature review and validity evaluation of the expanded disability status scale (EDSS) and the multiple sclerosis functional composite (MSFC) in patients with multiple sclerosis. BMC Neurol. 2014; 14:58-2377-14-58. [PubMed: 24666846]

15. Kurtzke JF. Rating neurologic impairment in multiple sclerosis: An expanded disability status scale (EDSS). Neurology. 1983; 33(11):1444-1452. [PubMed: 6685237]

16. Lamers I, Feys P. Assessing upper limb function in multiple sclerosis. Mult Scler. 2014

17. Brissart H, Sauvee M, Latarche C, Dillier C, Debouverie M. Integration of cognitive impairment in the expanded disability status scale of 215 patients with multiple sclerosis. Eur Neurol. 2010; 64(6):345-350. [PubMed: 21071951]

18. Kragt JJ, Thompson AJ, Montalban X, et al. Responsiveness and predictive value of EDSS and MSFC in primary progressive MS. Neurology. 2008; 70(13 Pt 2):1084-1091. [PubMed: 18184917]

19. Rudick RA, Polman CH, Cohen JA, et al. Assessing disability progression with the multiple sclerosis functional composite. Mult Scler. 2009; 15(8):984-997. [PubMed: 19667023]

*20. Rudick RA, Larocca N, Hudson LD. MSOAC. Multiple sclerosis outcome assessments consortium: Genesis and initial project plan. Mult Scler. 2014; 20(1):12-17. Describes the blueprint for an academic industry and regulatory effort for development of clinical trial outcome measures in MS. [PubMed: 24057430]

21. Ontaneda D, Larocca N, Coetzee T, Rudick R. Revisiting the multiple sclerosis functional composite: Proceedings from the national multiple sclerosis society (NMSS) task force on clinical disability measures. Mult Scler. 2012

22. Hawker K, O’Connor P, Freedman MS, et al. Rituximab in patients with primary progressive multiple sclerosis: Results of a randomized double-blind placebo-controlled multicenter trial. Ann Neurol. 2009; 66(4):460-471. [PubMed: 19847908]

23. Suhy J, Rooney WD, Goodkin DE, et al. 1H MRSI comparison of white matter and lesions in primary progressive and relapsing-remitting MS. Mult Scler. 2000; 6(3):148-155. [PubMed: 10871825]

24. Losseff NA, Wang L, Lai HM, et al. Progressive cerebral atrophy in multiple sclerosis. A serial MRI study. Brain. 1996; 119(Pt 6):2009-2019. [PubMed: 9010005]

25. Pagani E, Rocca MA, Gallo A, et al. Regional brain atrophy evolves differently in patients with multiple sclerosis according to clinical phenotype. AJNR Am J Neuroradiol. 2005; 26(2):341-346. [PubMed: 15709132]

26. Bo L, Vedeler CA, Nyland HI, Trapp BD, Mork SJ. Subpial demyelination in the cerebral cortex of multiple sclerosis patients. J Neuropathol Exp Neurol. 2003; 62(7):723-732. [PubMed: 12901699] 
27. Stevenson VL, Leary SM, Losseff NA, et al. Spinal cord atrophy and disability in MS: A longitudinal study. Neurology. 1998; 51(1):234-238. [PubMed: 9674808]

28. Schlaeger R, Papinutto N, Panara V, et al. Spinal cord gray matter atrophy correlates with multiple sclerosis disability. Ann Neurol. 2014; 76(4):568-580. [PubMed: 25087920]

29. Agosta F, Absinta M, Sormani MP, et al. In vivo assessment of cervical cord damage in MS patients: A longitudinal diffusion tensor MRI study. Brain. 2007; 130(Pt 8):2211-2219. [PubMed: 17535835]

30. Pulicken M, Gordon-Lipkin E, Balcer LJ, Frohman E, Cutter G, Calabresi PA. Optical coherence tomography and disease subtype in multiple sclerosis. Neurology. 2007; 69(22):2085-2092. [PubMed: 18040015]

31. Panitch H, Goodin DS, Francis G, et al. Randomized, comparative study of interferon beta-1a treatment regimens in MS: The EVIDENCE trial. Neurology. 2002; 59(10):1496-1506. [PubMed: 12451188]

32. Andersen O, Elovaara I, Farkkila M, et al. Multicentre, randomised, double blind, placebo controlled, phase III study of weekly, low dose, subcutaneous interferon beta-1a in secondary progressive multiple sclerosis. J Neurol Neurosurg Psychiatry. 2004; 75(5):706-710. [PubMed: 15090564]

33. Cohen JA, Cutter GR, Fischer JS, et al. Benefit of interferon beta-1a on MSFC progression in secondary progressive MS. Neurology. 2002; 59(5):679-687. [PubMed: 12221157]

34. Secondary Progressive Efficacy Clinical Trial of Recombinant Interferon-Beta-1a in MS (SPECTRIMS) Study Group. Randomized controlled trial of interferon- beta-1a in secondary progressive MS: Clinical results. Neurology. 2001; 56(11):1496-1504. [PubMed: 11402106]

35. European Study Group on interferon beta-1b in secondary progressive MS. Placebo-controlled multicentre randomised trial of interferon beta- $1 \mathrm{~b}$ in treatment of secondary progressive multiple sclerosis. Lancet. 1998; 352(9139):1491-1497. [PubMed: 9820296]

36. Wolinsky JS, Narayana PA, O'Connor P, et al. Glatiramer acetate in primary progressive multiple sclerosis: Results of a multinational, multicenter, double-blind, placebo-controlled trial. Ann Neurol. 2007; 61(1):14-24. [PubMed: 17262850]

*37. [Accessed 12/11/2014] Novartis provides update on fingolimod phase III trial in primary progressive MS (PPMS). 2014. http://www.novartis.com/newsroom/media-releases/en/ 2014/1875463.shtmlResults of a large negative phase III trial of fingolimod in PPMS

38. Kappos L, Weinshenker B, Pozzilli C, et al. Interferon beta-1b in secondary progressive MS: A combined analysis of the two trials. Neurology. 2004; 63(10):1779-1787. [PubMed: 15557490]

39. British And Dutch Multiple Sclerosis Azathioprine Trial Group. Double-masked trial of azathioprine in multiple sclerosis. british and dutch multiple sclerosis azathioprine trial group. Lancet. 1988; 2(8604):179-183. [PubMed: 2899660]

40. The Multiple Sclerosis Study Group. Efficacy and toxicity of cyclosporine in chronic progressive multiple sclerosis: A randomized, double-blinded, placebo-controlled clinical trial. Ann Neurol. 1990; 27(6):591-605. [PubMed: 2193613]

41. The Canadian Cooperative Multiple Sclerosis Study Group. The canadian cooperative trial of cyclophosphamide and plasma exchange in progressive multiple sclerosis. Lancet. 1991; 337(8739):441-446. [PubMed: 1671468]

42. Goodkin DE, Rudick RA, VanderBrug Medendorp S, et al. Low-dose (7.5 mg) oral methotrexate reduces the rate of progression in chronic progressive multiple sclerosis. Ann Neurol. 1995; 37(1): 30-40. [PubMed: 7818255]

**43. Chataway J, Schuerer N, Alsanousi A, et al. Effect of high-dose simvastatin on brain atrophy and disability in secondary progressive multiple sclerosis (MS-STAT): A randomised, placebocontrolled, phase 2 trial. Lancet. 2014 A large phase IIb trial demonstrating a significant effect of simvastatin on brain atrophy in SPMS.

44. Kapoor R, Furby J, Hayton T, et al. Lamotrigine for neuroprotection in secondary progressive multiple sclerosis: A randomised, double-blind, placebo-controlled, parallel-group trial. Lancet Neurol. 2010; 9(7):681-688. [PubMed: 20621711]

*45. Zajicek J, Ball S, Wright D, et al. Effect of dronabinol on progression in progressive multiple sclerosis (CUPID): A randomised, placebo-controlled trial. Lancet Neurol. 2013; 12(9):857-865. 
An example of a large negative phase III trial where there was a low proportion of progression and highlighting the need for phase II trials prior to phase III studies. [PubMed: 23856559]

46. Palace J, Robertson N. Modifying disability in progressive multiple sclerosis. Lancet. 2014; 383(9936):2189-2191. [PubMed: 24655728]

47. Vermersch P, Benrabah R, Schmidt N, et al. Masitinib treatment in patients with progressive multiple sclerosis: A randomized pilot study. BMC Neurol. 2012; 12:36-2377-12-36. [PubMed: 22691628]

48. Barkhof F, Hulst HE, Drulovic J, et al. Ibudilast in relapsing-remitting multiple sclerosis: A neuroprotectant? Neurology. 2010; 74(13):1033-1040. [PubMed: 20200338]

**49. [Accessed 5/6/2014, 2014] Safety, tolerability and activity study of ibudilast in subjects with progressive multiple sclerosis - full text view. ClinicalTrials.gov. http://clinicaltrials.gov/ct2/ show/NCT01982942National Institutes of Health sponsored multi-center phase II trial of ibudilast in progressive forms of MS

**50. MS-SMART. [Accessed 12/11/2014] Multiple sclerosis-secondary progressive multi-arm randomisation trial - full text view - ClinicalTrials.gov. 2014. http://clinicaltrials.gov/ct2/show/ NCT01910259Large multi-arm phase II trial of several neuroprotectant agents in secondary progressive MS

51. Denic A, Johnson AJ, Bieber AJ, Warrington AE, Rodriguez M, Pirko I. The relevance of animal models in multiple sclerosis research. Pathophysiology. 2011; 18(1):21-29. [PubMed: 20537877]

52. Chataway J, Nicholas R, Todd S, et al. A novel adaptive design strategy increases the efficiency of clinical trials in secondary progressive multiple sclerosis. Mult Scler. 2011; 17(1):81-88.

[PubMed: 20798135]

*53. [Accessed 12/12/2014, 2014] International progressive MS alliance. http:// www.progressivemsalliance.org/International effort for forwarding scientific understanding and therapeutics development in progressive forms of MS 


\section{Key Points}

1. The pathogenesis of PMS remains poorly understood, but this stage of the disease appears to have a combination of inflammatory features with a significant neurodegenerative component.

2. Clinical trial results in PMS have been disappointing to date and antiinflammatory agents have not shown significant effects.

3. Many therapies are currently in clinical development and testing and hold great promise.

4. Refining clinical outcome measures, imaging measures and clinical trial design will aid in accelerating and streamlining the development of PMS therapeutics.

5. International efforts including the Progressive MS Alliance and the Multiple Sclerosis Outcomes and Assessment Consortium are currently underway to accelerate treatment discovery for PMS. 

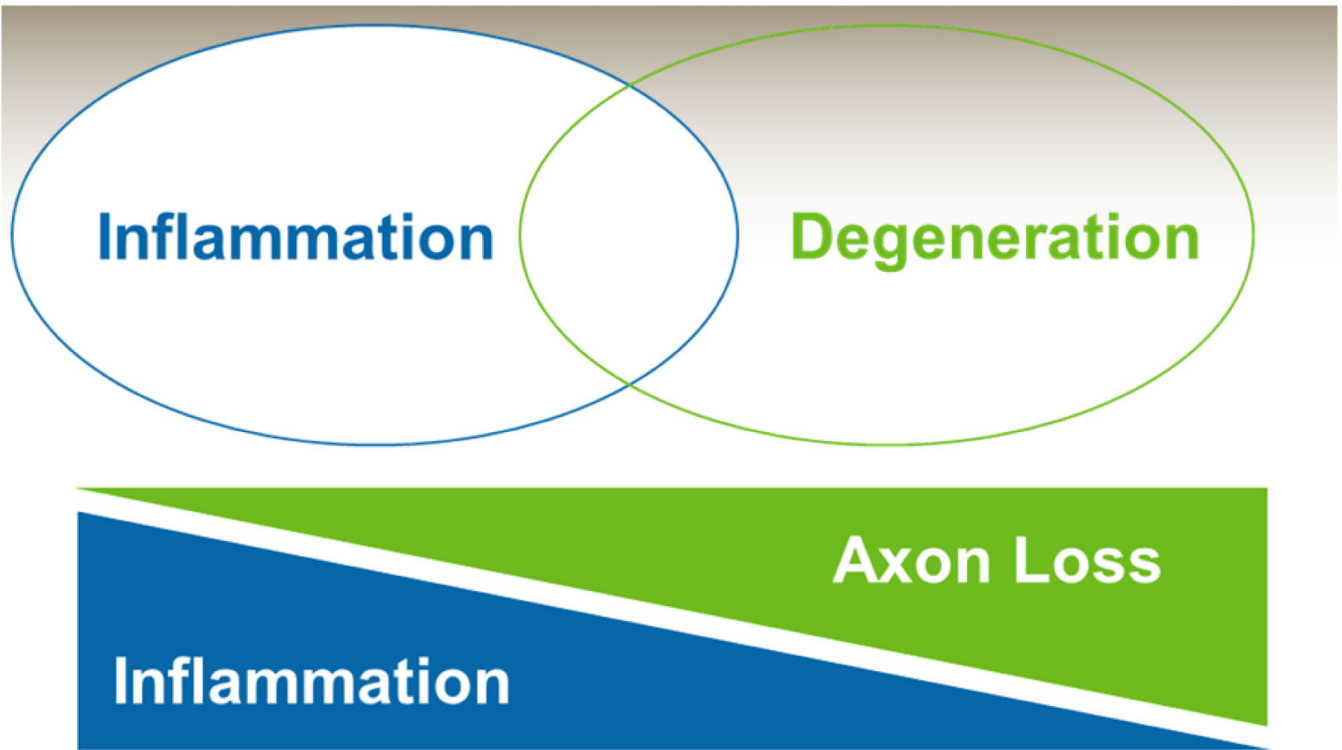

RR

Figure 1.

Degenerative and inflammatory components in PMS

Relapsing remitting (RR), secondary progressive (SP)

Source: Authors 


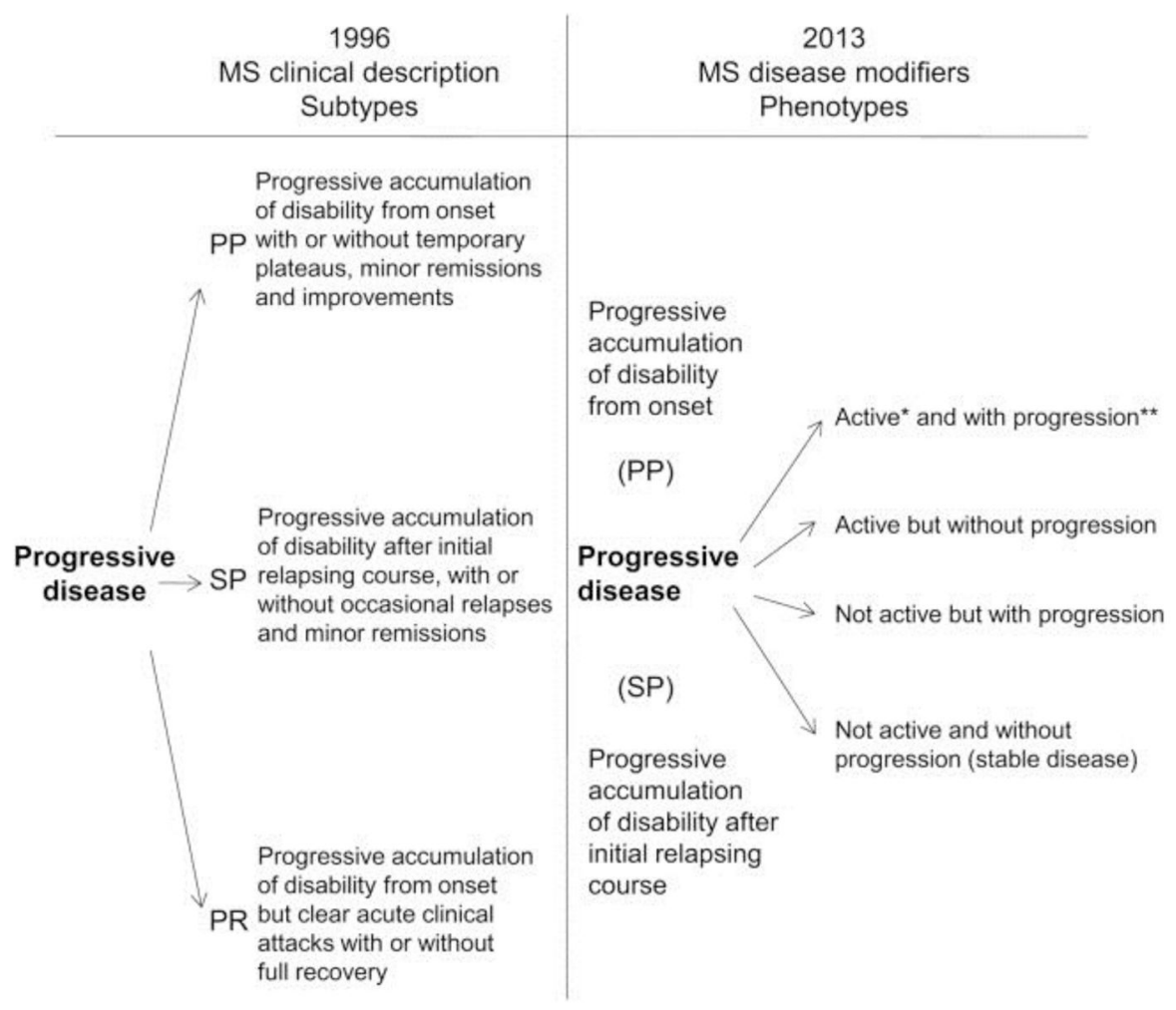

Figure 2.

Proposed Classification of Clinical Course in PMS

Taken from:

Lublin FD, Reingold SC, Cohen JA, et al. Defining the clinical course of multiple sclerosis: The 2013 revisions. Neurology. 2014;83(3):278-286. 
Table 1

Ongoing treatment trials in PMS, phase III and selected phase II studies

\begin{tabular}{|l|c|c|c|c|}
\hline Agent & Disease Course & Duration & Trial Size & Phase \\
\hline Amiloride (MS-SMART) & SPMS & 24 months & 440 (divided among 4 arms) & II \\
\hline Ibudilast (SPRINT MS) & PP, SPMS & 24 months & 250 & II \\
\hline Ibudilast (MS-SMART) & SPMS & 24 months & 440 (divided among 4 arms) & II \\
\hline Idebonone & SPMS & 24 months & 80 & II \\
\hline Imilecleucel-T & SPMS & 24 months & 180 & II \\
\hline Masitinib & PP, SPMS & 24 months & 450 & III \\
\hline Natalizumab & SPMS & 24 months & 856 & III \\
\hline Ocrelizumab & PPMS & 24 months & 733 & III \\
\hline Riluzole (MS-SMART) & SPMS & 25 months & 440 (divided among 4 arms) & II \\
\hline Spinonimod & SPMS & 40 months & 1530 & III \\
\hline T Cell Receptor Peptide & SPMS & 12 months & 200 & II \\
\hline
\end{tabular}

Source: Authors, data obtained from clinicaltrials.gov 